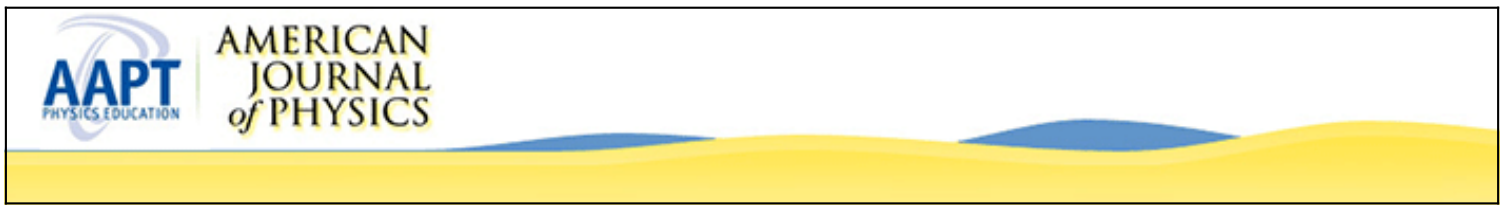

\title{
Variational treatment of the linear potential
}

B. Cameron Reed

Citation: American Journal of Physics 58, 407 (1990); doi: 10.1119/1.16460

View online: http://dx.doi.org/10.1119/1.16460

View Table of Contents: http://scitation.aip.org/content/aapt/journal/ajp/58/4?ver=pdfcov

Published by the American Association of Physics Teachers

\section{Articles you may be interested in}

Effective potential methods in variational treatments of electron-molecule collisions. II. Application to $\mathrm{HBr}$

J. Chem. Phys. 104, 125 (1996); 10.1063/1.470882

Effective potential methods in variational treatments of electron-molecule collisions. I. Theoretical formulation

J. Chem. Phys. 104, 120 (1996); 10.1063/1.470881

Comment on "Variational treatment of the linear potential," by B. Cameron Reed [Am. J. Phys. 58 , 407-408 (1990)]

Am. J. Phys. 59, 852 (1991); 10.1119/1.16736

Variational-perturbational treatment for the polarizabilities of conjugated chains. I. Theory and linearpolarizabilities results for polyenes

J. Chem. Phys. 88, 2558 (1988); 10.1063/1.454035

Parameter Variations in Linear Memories

J. Acoust. Soc. Am. 47, 125 (1970); 10.1121/1.1974040

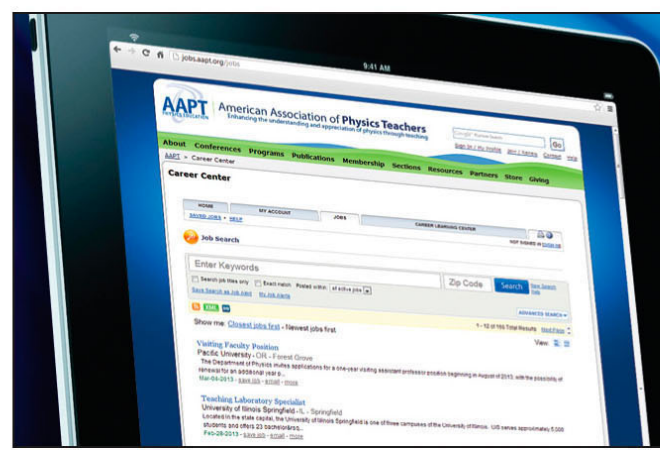

American Association of Physics Teachers

Explore the AAPT Career Center access hundreds of physics education and other STEM teaching jobs at two-year and four-year colleges and universities.

\section{http://jobs.aapt.org}


aperture of a single slit and the visibility of the interference pattern in Young's experiment. Furthermore, it is worth noting that an image-processing package can be used to get quantitative results in other optics and spectroscopy experiments like, for instance, "study of the theory of diffraction," "Fourier optics and spatial filter and correlation,", "Isotope effect," and "Zeeman effect."

\section{ACKNOWLEDGMENTS}

This project has been one of many in a long-term effort to modernize the advanced laboratory in our department. Many students and technical staffs have contributed to this effort in the past. We express our deep gratitude to everyone who has been involved in this effort. Many thanks are due to Professor G. J. Jan and K. T. Hsu for their advice.
Work supported by the National Science Council of the Republic of China, NSC 76-011-S002-10.

'See, for instance, Eugene Hecht, Optics (Addison-Wesley, Reading, MA, 1975), 2nd ed., Chap. 10.

'The lab experiment was originally set up (photographic method) by J. C. Hung and the detailed lab manual (in Chinese) was written by him and has been used for more than 3 years.

'Model VID-512 Frame Grabber, product of Vision Inc., 34 East 4th Industrial Rd. Science-based Industrial Park, Hsinchu, Taiwan, R. O. C. ${ }^{4}$ F. T. Yu and E. Y. Wang, "Undergraduate coherent optics laboratory," Am. J. Phys. 41, 1160-1169 (1973).

D. Bloor, "Coherence and correlation-Two advanced experiments in optics," Am. J. Phys. 32, 936-1941 (1964).

"S. George, "Hyperfine structure in Bismuth-An experiment," Am. J. Phys. 36, 27-29 (1968).

\title{
Variational treatment of the linear potential
}

\author{
B. Cameron Reed \\ Department of Physics, Saint Mary's University, Halifax, Nova Scotia B3H 3C3, Canada
}

(Received 9 November 1988; accepted for publication 10 April 1989)

In a recent note, Lee' presented two examples of the use of the variational method: to a harmonic oscillator potential and to a screened Yukawa potential. The first of these is somewhat of a null problem in that a judicious choice of the trial wavefunction leads to the exact solution, obscuring the point of showing how the technique is used to give limits on the ground-state energy. The second example is complicated by uncertain knowledge of the screening parameter, and can, in fact, be found as an example in a well-known text. ${ }^{2}$ The purpose of this note is to describe an example of the variational method I have found useful in a junior level introductory quantum physics course, that of a one-dimensional linear potential, $V(x)=\alpha x$. This problem is easy to postulate a trial wavefunction for, and is tractable analytically. In addition, as it is easily addressed with the WKB method, students have an opportunity to compare the merits of various approximation schemes.

The essence of the variational method is as follows. If $\phi(x)$ is a normalized trial wavefunction for the ground state of a system, then the ground-state energy $E_{0}$ satisfies $^{3}$

$$
E+\sqrt{D-E^{2}} \geqslant E_{0} \geqslant E-\sqrt{D-E^{2}}
$$

where

$$
\begin{aligned}
& E=\int \phi^{*} \phi d x, \\
& D=\int(H \phi)^{*}(H \phi) d x,
\end{aligned}
$$

with

$$
H=-\epsilon \frac{d^{2}}{d x^{2}}+V(x),
$$

where $\epsilon=\hbar^{2} / 2 m$. Any arbitrary chosen trial wavefunction is acceptable as long as it is normalized and satisfies the boundary conditions of the problem. In practice, one sets up some trial wavefunction with one or more adjustable parameters and varies the parameters to minimize $\left(D-E^{2}\right)$.

Figure 1 illustrates the situation for the linear potential. From the usual rules for sketching wavefunctions, ${ }^{4}$ we can assume that a plausible ground-state wavefunction must have one maximum followed by the exponential decay characteristic of tunneling phenomena. A simple function that meets these requirements and satisfies the boundary conditions is

$$
\phi(x)=A x \exp (-\beta x) .
$$

Normalizing between $x=0$ and $x=\infty$ gives

$$
A=2 \beta^{3 / 2} \text {. }
$$

From Eqs. (2)-(4) we find, after some algebra,

$$
E=\epsilon \beta^{2}+3 \alpha / 2 \beta
$$

and

$$
D=5 \epsilon^{2} \beta^{4}+\alpha \epsilon \beta+3 \alpha^{2} / \beta^{2} .
$$

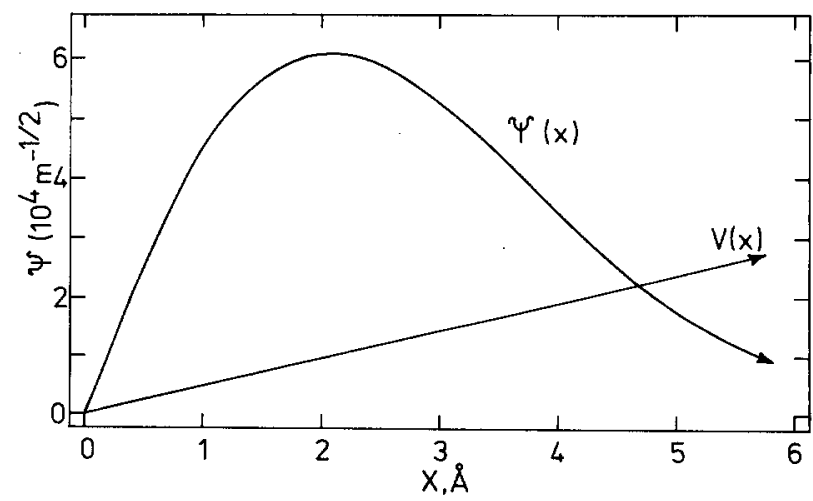

Fig. 1. Plausible ground-state wavefunction for the linear potential. See discussion following Eq. (23). The $V(x)$ curve is schematic only. 


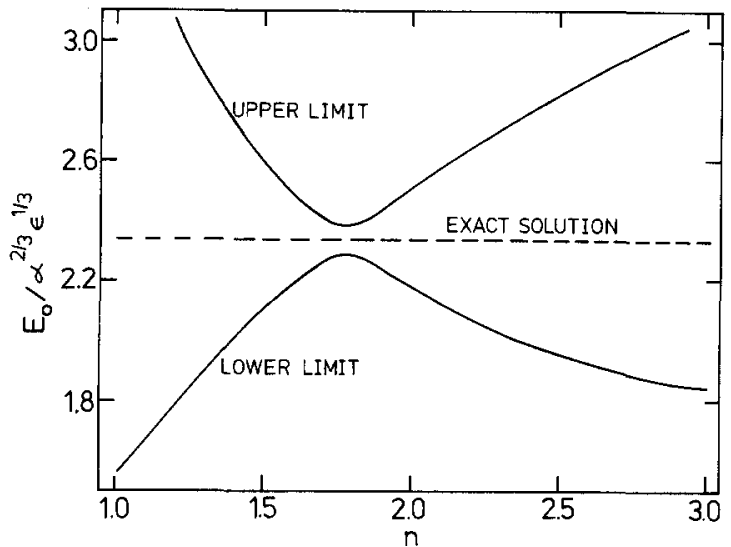

Fig. 2. Upper and lower limits on the ground-state wavefunction of Eq. (13) as a function of $n$. The dashed line gives the exact solution.

Minimizing $D-E^{2}$ with respect to $\beta$ yields a quadratic equation in $\beta^{3}$,

$$
16 \epsilon^{2} \beta^{6}-2 \epsilon \alpha \beta-3 \alpha^{2} / 2=0,
$$

which has roots

$$
\beta^{3}=3 \alpha / 8 \epsilon \text { or }-\alpha / 4 \epsilon \text {. }
$$

The second root in Eq. (9) implies a complex (hence, unphysical) energy, which we reject. The real root gives

$$
\begin{aligned}
\sqrt{\left(D-E^{2}\right)_{\min }} & =2(3 / 8)^{2 / 3} \alpha^{2 / 3} \epsilon^{1 / 3} \\
& =1.040 \alpha^{2 / 3} \epsilon^{1 / 3}
\end{aligned}
$$

and

$$
E_{\min }=5(3 / 8)^{2 / 3} \alpha^{2 / 3} \epsilon^{1 / 3}=2.600 \alpha^{2 / 3} \epsilon^{1 / 3} .
$$

Hence, we find

$$
3.640 \geqslant\left(E_{0} / \alpha^{2 / 3} \epsilon^{1 / 3}\right) \geqslant 1.560 \text {. }
$$

This result brackets almost perfectly the exact solution, $\left(E_{0} / \alpha^{2 / 3} \epsilon^{1 / 3}\right)=2.338$. A WKB analysis gives $\left(E_{0} /\right.$ $\left.\alpha^{2 / 3} \epsilon^{1 / 3}\right) \approx 2.811$.

A more challenging problem is given by modifying Eq. (5) to the case of a generalized exponential decay,

$$
\phi(x)=A x \exp \left(-\beta x^{n}\right) .
$$

The exponent $n$ is presumed fixed; $\beta$ will be varied. The normalization constant is given by

$$
A^{2}=n(2 \beta)^{3 / n} / \Gamma(3 / n) \text {. }
$$

It is straightforward, if tedious, to show that minimizing $\left(D-E^{2}\right)$ with respect to $\beta$ leads to a quadratic equation in $\beta^{3 / n}$ with one positive root:

$$
\beta_{\min }^{3 / n}=\left(\frac{\sqrt{K^{2}-32 L J}-K}{8 L}\right)\left(\frac{\alpha}{\epsilon}\right)=\eta\left(\frac{\alpha}{\epsilon}\right),
$$

where $J, K$, and $L$ are given by

$$
\left.\begin{array}{l}
J=F-B^{2}, \\
K=G-2 B C, \\
L=H-C^{2},
\end{array}\right\}
$$

where $B, C, F, G$, and $H$ are given by

$$
\begin{aligned}
B= & \Gamma(4 / n) / 2^{1 / n} \Gamma(3 / n) \\
C= & \frac{2^{2 / n}}{\Gamma(3 / n)}\left[\frac{1}{2} n(n+1) \Gamma\left(\frac{n+1}{n}\right)\right. \\
& \left.-\frac{1}{4} n^{2} \Gamma\left(\frac{2 n+1}{n}\right)\right] \\
F= & \Gamma(5 / n) / 2^{2 / n} \Gamma(3 / n) \\
G= & \frac{2^{1 / n}}{\Gamma(3 / n)}\left[n(n+1) \Gamma\left(\frac{n+2}{n}\right)\right. \\
& \left.-\frac{1}{2} n^{2} \Gamma\left(\frac{2 n+2}{n}\right)\right], \\
H= & \frac{1}{4} \frac{n^{2} 2^{4 / n}}{\Gamma(3 / n)}\left[(1+n)^{2} \Gamma\left(\frac{2 n-1}{n}\right)\right. \\
& \left.-n(1+n) \Gamma\left(\frac{3 n-1}{n}\right)+\frac{1}{4} n^{2} \Gamma\left(\frac{4 n-1}{n}\right)\right] .
\end{aligned}
$$

With these results we find

$$
\sqrt{\left(D-E^{2}\right)_{\min }}=\eta^{-1 / 3} \sqrt{J+K \eta+L \eta^{2}}\left(\alpha^{2 / 3} \epsilon^{1 / 3}\right)
$$

and

$$
E_{\min }=\eta^{-1 / 3}(B+C \eta)\left(\alpha^{2 / 3} \epsilon^{1 / 3}\right) .
$$

Upper and lower limits for $E_{0}$ as a function of $n$ are shown in Fig. 2. The most stringent limit obtains when $n \approx 1.773$, where $2.378 \geqslant\left(E_{0} / \alpha^{2 / 3} \epsilon^{1 / 3}\right) \geqslant 2.298$, which brackets the exact result to better than $2 \%$. The wavefunction plotted in Fig. 1 corresponds to this value of $n$ for an electron in a potential with $\alpha=1 \mathrm{eV} / \AA$.

'Johnson Lee, "The upper and lower bounds of the ground state energies using the variational method," Am. J. Phys. 55, 1039-1040 (1987).

${ }^{2}$ J. L. Powell and B. Craseman, Quantum Mechanics (Addison-Wesley, Reading, MA, 1961), p. 398.

${ }^{3}$ Linus Pauling and E. Bright Wilson, Jr., Introduction to Quantum Mechanics (McGraw-Hill, New York, 1935), Chap. 7.

${ }^{4}$ A. P. French and Edwin F. Taylor, An Introduction to Quantum Physics (Norton, New York, 1978), Chap. 3.

\title{
Maximum ranges in ideal projectile motion-A generalization
}

\author{
Drago Bajc \\ Department of Education, The University of Maribor, Maribor, Slovenia, Yugoslavia
}

(Received 29 January 1988; accepted for publication 9 April 1989)

The maximum range of a projectile shot from a given height over a horizontal plane, or from a point of an inclined plane, as well as the maximum height attained on a vertical wall, have all received ample discussion in this Journal, the emphasis having been placed mostly on elementary solutions requiring no calculus (see, for example, 